University of Nebraska - Lincoln

DigitalCommons@University of Nebraska - Lincoln

USDA Wildlife Services - Staff Publications

U.S. Department of Agriculture: Animal and Plant Health Inspection Service

2018

\title{
Quantifying site-level usage and certainty of absence for an invasive species through occupancy analysis of camera-trap data
}

Amy J. Davis

USDA National Wildlife Research Center, Amy.J.Davis@usda.gov

Ryan McCreary

USDA APHIS Wildlife Services

Jeremiah Psiropoulos

USDA APHIS Wildlife Services

Gary Brennan

USDA APHIS Wildlife Services

Terry Cox

USDA APHIS Wildlife Services

See next page for additional authors

Follow this and additional works at: https://digitalcommons.unl.edu/icwdm_usdanwrc

Part of the Life Sciences Commons

Davis, Amy J.; McCreary, Ryan; Psiropoulos, Jeremiah; Brennan, Gary; Cox, Terry; Partin, Andrew; and Pepin, Kim M., "Quantifying site-level usage and certainty of absence for an invasive species through occupancy analysis of camera-trap data" (2018). USDA Wildlife Services - Staff Publications. 2077.

https://digitalcommons.unl.edu/icwdm_usdanwrc/2077

This Article is brought to you for free and open access by the U.S. Department of Agriculture: Animal and Plant Health Inspection Service at DigitalCommons@University of Nebraska - Lincoln. It has been accepted for inclusion in USDA Wildlife Services - Staff Publications by an authorized administrator of DigitalCommons@University of Nebraska - Lincoln. 


\section{Authors}

Amy J. Davis, Ryan McCreary, Jeremiah Psiropoulos, Gary Brennan, Terry Cox, Andrew Partin, and Kim M. Pepin 


\title{
Quantifying site-level usage and certainty of absence for an invasive species through occupancy analysis of camera-trap data
}

\author{
Amy J. Davis $(\mathbb{D} \cdot$ Ryan McCreary $\cdot$ Jeremiah Psiropoulos • Gary Brennan • \\ Terry Cox $\cdot$ Andrew Partin $\cdot$ Kim M. Pepin
}

Received: 23 November 2016/Accepted: 9 October 2017/Published online: 13 October 2017

(C) Springer International Publishing AG (outside the USA) 2017

This document is a U.S. government work and is not subject to copyright in the United States.

\begin{abstract}
Efficient implementation of management programs for invasive species depends on accurate surveillance for guiding prioritization of surveillance and control resources in space and time. Occupancy probabilities can be used to determine where surveillance should occur. Conversely, knowledge of the certainty of site-level absence is of special interest in situations where the objective is to completely remove populations despite substantial risk of reinvasion. Indeed, the decision to shift from emphasizing control activities over the full range to emphasizing reinvasion prevention, surveillance, and response near the borders, depends on accurate knowledge of absence across space. We used a dynamic occupancy model to monitor changes in the distribution of an invasive species, feral swine (Sus scrofa), based on camera-trap data collected as part of a management program from June 2014 to January 2016 in San Diego County, California. Site usage of feral swine declined
\end{abstract}

Electronic supplementary material The online version of this article (doi:10.1007/s10530-017-1579-x) contains supplementary material, which is available to authorized users.

A. J. Davis $(\square) \cdot$ K. M. Pepin

National Wildlife Research Center, USDA, APHIS,

Fort Collins, CO 80521, USA

e-mail: Amy.J.Davis@aphis.usda.gov

R. McCreary · J. Psiropoulos · G. Brennan ·

T. Cox $\cdot$ A. Partin

Wildlife Services, USDA, APHIS, El Cajon, CA, USA overall. The most informative predictors of site usage were spatial (latitude and longitude). Site-level nonusage rates increased over time and in response to management removal efforts; and site-level usage rates were heavily impacted by having neighboring sites that were used. Combining the detection probability estimated from the occupancy model and Bayes Theorem, we demonstrated how certainty of local (site-level) absence can be estimated iteratively in time in areas with negative surveillance (no detections) data. Our framework provides a means for using management-based surveillance data to quantify certainty of site-level absence of an invasive species, allowing for adaptive prioritization of surveillance and control resources. Our approach is flexible for application to other species and types of surveillance (e.g., track-plates, eDNA).

Keywords Camera trap - Dynamic occupancy . Elimination - Eradication - Invasive species ·

Surveillance $\cdot$ Sus scrofa

\section{Introduction}

Wildlife managers are often tasked with protecting resources in a defined area by reducing invasive species populations, completely when possible, despite a substantial risk of reinvasion from neighboring populations (Edge et al. 2011; Myers et al. 
2000; Parkes et al. 2017), which precludes eradication (Bomford and O'Brien 1995). When the objective of management is complete removal, but there is reinvasion potential, the terminology for invasive species work ranges from control (Allendorf and Lundquist 2003), area-wide suppression (Myers et al. 2000), complete removal (Robertson et al. 2016), to extirpation (Edge et al. 2011; Parkes et al. 2017). Using the term 'control' does not distinguish between objectives where complete removal is desired versus some other intensity of removal. Management and spread of invasive species are similar to that of emerging infectious diseases (Crowl et al. 2008), for which there is a clear set of definitions for levels of control in populations with reinvasion risk (Dowdle 1998). Adapted for invasive species, these terms are (also see Fig. 1).

- Sustained control: reduction of invasive species to a locally acceptable level as a result of control; continued control measures are required to maintain reduced populations.

- Elimination: reduction to zero of the invasive species in a defined geographical area as a result of control; continued prevention, surveillance, and control of reinvaders is needed.

- Eradication: reduction to zero of the invasive species in a defined geographical area as a result of control with little or no chance of reinvasion (as per Bomford and O'Brien 1995).

If natural borders, or funding to create artificial borders, do not exist then eradication will not be possible and reduction of invasive species will fall into either sustained control or elimination categories. When areas are at the boundaries of new invasions it may be preferable to focus on elimination instead of sustained control to prevent future spread of the invasion. Additionally, in areas with low densities and low immigration rates elimination may be more efficient than sustained control. Elimination must be done through a two-step process: (1) removal of the population in the target area (maximum control; Fig. 1b), and (2) ongoing early detection and rapid response (EDRR) to reinvasions (perpetual EDRR; Fig. 1b). There are many studies that explore the challenges with the EDRR step of this process, for example: how to implement monitoring for negative surveillance under resource limitations, what are the cost trade-offs for implementing surveillance (spend money now) versus risking reinvasions (don't spend money now but risk spending money for damage recovery and control later), and what strategies for rapid response programs are most effective for the invasive species of interest (e.g., Rabaglia et al. 2008; Simpson et al. 2009; Westbrooks 2004). Determining there is a high probability of elimination in most of the

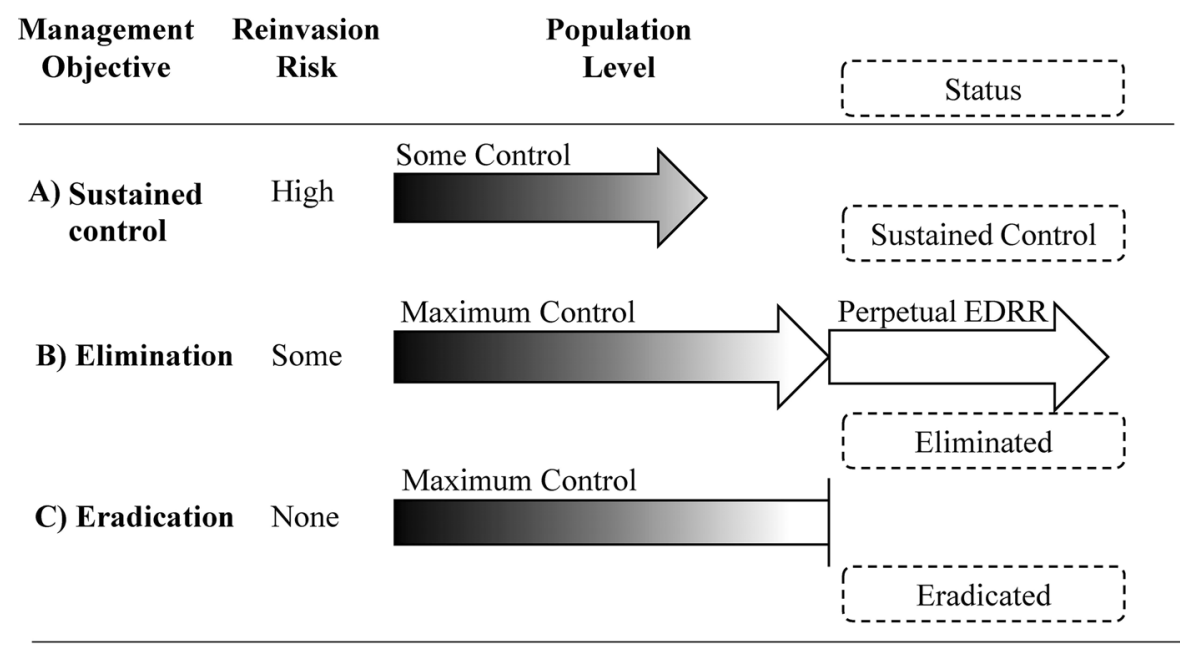

Fig. 1 Schematic showing the distinction between management objections given different reinvasion risks. The darkness of the arrows represent the population level (dark indicates higher density, white represents no population). The management action is perpetual for objectives $A$ and $B$. The management actions change from maximum control to perpetual early detection and rapid response (EDRR) for elimination objective $(B)$, but management ends when eradication is successful for the eradication objective $(C)$. The resulting ideal status is shown by objective in dashed boxes 
study area is critical before addressing other challenges of EDRR programs.

Elimination programs (Fig. 1b) require an accurate assessment of the status of the invasive species in the area, a targeted effort to remove the species, and continued surveillance to determine success of elimination efforts. Surveillance is a critical element to effective management regardless of the objectives, but particularly for successful programs where the objective is complete removal (Anderson et al. 2013; Lyons et al. 2008). An inherent challenge with quantifying the certainty a species is truly eliminated from an area is knowing when lack of detection indicates true absence (Anderson et al. 2013). Therefore quantifying detection probability given a species is present is an important quantity for evaluating the certainty of absence (MacKenzie 2005; Ramsey et al. 2009; Regan et al. 2006; Rout et al. 2014). Detection probabilities are notoriously low as populations diminish (e.g., MacKenzie et al. 2005), requiring more intensive surveillance to be certain of elimination. If detection is low, either due to minimal surveillance or poor allocation of efforts, the target species may have a chance to persist and rebound (Bomford and O'Brien 1995; Regan et al. 2006). One way to increase detection at low densities within budget constraints is to use an adaptive surveillance plan within the larger study area, where sites (e.g., gridded partitions of the study area) with high probability of absence are prioritized for resource allocation compared with sites with low probability of absence. Specifically, surveillance data can be analyzed to determine the certainty of site-level absence across the area of interest in order to guide allocation of surveillance and control resources as the system changes in time.

There are many methods for surveillance wildlife populations for example aerial surveys, animal marking methods, removal sampling, camera traps, track plots, etc. (Engeman et al. 2013; O'Connell et al. 2010). However, some analytical methods for estimating population metrics (e.g., density, abundance) and quantifying associated detection rates are not well suited for low density populations, for example, removal modeling (Davis et al. 2016) and capture/recapture methods (Seber 1982). Other methods such as occupancy analysis using passive detectors like camera traps are commonly used for low-density species (MacKenzie et al. 2006). Camera traps can be particularly useful for managers as cameras give time and date information which can guide managers in targeting individuals for removal.

Occupancy analysis is becoming increasingly common as a management tool for surveillance wildlife populations (MacKenzie et al. 2009), and is well suited to camera trap data (Shannon et al. 2014; Thorn et al. 2009; Tobler et al. 2015). Occupancy is estimated from presence/absence data while accounting for imperfect detection (MacKenzie et al. 2009). Dynamic occupancy models evaluate patterns in occupancy status across time, determine factors related to local extinctions (occupied sites that become unoccupied), colonizations (unoccupied sites that become occupied), and detection (probability of detecting an individual given the site is occupied). Occupancy analysis assumes that the sites are closed to changes in the occupancy status during the study (MacKenzie et al. 2006). When closure assumptions are relaxed, occupancy can be thought of as the probability of site 'usage' (Kendall et al. 2013). Thus the definitions in dynamic occupancy change slightly: local extinctions are used sites becoming unused, local colonizations are unused sites that become used, and detection is the probability of detecting a species given the site was used. The occupancy framework can be used to determine the conditional probability of nonsite-usage ('certainty of absence') given sampling effort by a species (MacKenzie et al. 2006). Using occupancy models and probability theory we can determine the certainty within the larger study area, of site-level absence from recent negative surveillance data iteratively, based on the current effort employed and site-level probability of non-usage, allowing adaptive prioritization of surveillance resources.

The objectives of our study were to (1) quantify changes in site (i.e. gridded partitions of the study area) usage of an invasive species and factors driving changes over time, (2) evaluate effectiveness of management actions on site-level usage, (3) identify priority areas for increased surveillance and removal while the invasive species is still present in the study area, and (4) demonstrate how our approach could be adapted to estimate a spatial surface for the certainty of site-level absence through time, by iteratively updating estimates with the most recent surveillance data, and using results to guide resource allocation decisions adaptively. It is best to design surveillance studies to have random sampling, replication, and large sample sizes. However, this is often not feasible, 
especially for surveillance during large-scale elimination. To address these resource challenges, we applied an occupancy modeling framework to data collected by managers tasked with surveillance and controlling feral swine to demonstrate the utility of this approach to non-standard sampling designs. Our method could be easily adapted to determine elimination certainty for other species using other types of detectors.

\section{Study species and area}

Feral swine (Sus scrofa) are an invasive species in North America whose range has expanded in the United States (Bevins et al. 2014). Feral swine cause significant damage to natural resources, agriculture, private land, and endangered species (Roemer et al. 2002; Seward et al. 2004). In California there are a combination of free ranging domestic pigs interbred with Eurasian wild boar that were first released in Monterey County in 1925 (Hoehne 1994; Mayer and Brisbin 2008). Populations of feral swine have increased in California over the last three decades and they have expanded to 56 of 58 counties (California Department of Fish and Wildlife report). The study area comprised of $\sim 4500 \mathrm{~km}^{2}$ in San Diego County, California (Fig. 2). The area included federal land (BLM and Forest Service), state and city land (county parks and city of San Diego Public Utilities), and private property. Habitat in the study area consists of chaparral and riparian areas, oak woodland and grasslands in isolated areas, pasture, and pine forests at higher elevations. Elevations range from 152 to 1830 $\mathrm{m}$, however feral swine were most often detected at elevations of $380-1100 \mathrm{~m}$.

\section{Methods}

\section{Camera trapping}

Camera trapping was conducted in San Diego County, California from June 2014 thru January 2016. We placed 285 passive infrared camera traps on properties throughout the study area (Fig. 2). Camera placements were not randomly selected, but selected by managers to optimize spatial coverage in areas of concern for feral swine presence. To increase probability of detections, cameras were placed in areas with old feral swine sign (e.g., rooting, tracks, tree scars), travel corridors, water sources, suitable habitat, and where feral swine had been reported in the past. Camera placement was not limited by road access, if there were no roads in an area we hiked (on and off trail) to remote locations to place cameras and bait. We used Moultrie M880 IR trail cameras placed on trees or t-posts baited with corn mixed with yeast, dyed sugar mix, and water. The cameras were initially baited with approximately 15 pounds of bait and rebaited when needed. Cameras were motion activated and set to take 3 pictures immediately (no delay). Photographs were cataloged and the location of the camera, date and time of the picture, and number and identification of wild pigs were recorded in a spreadsheet.

\section{Management control activities}

In addition to camera trapping for surveillance feral swine, feral swine were removed in the area as part of the United States Department of Agriculture's (USDA) National Feral Swine Damage Management Program. Managers conducted removal events in target areas and in response to land owners requests. Removals were conducted either by ground shooting (conducted with dogs or using night vision equipment) or trapping (baited corral or cage traps, foot snares). Removals recorded in this study were conducted by USDA-Animal and Plant Health Inspection Service (APHIS)-Wildlife Services (WS) personnel who routinely provide wildlife-control assistance based on the authority of the Animal Damage Control Program of 1985 in compliance with the National Environmental Policy Act.

\section{Site-usage analysis}

Estimates of home range sizes for feral swine in California are near $2 \mathrm{~km}^{2}$ for females; male home ranges can be 50\% larger (Baber and Coblentz 1986; Saunders and McLeod 1999). Using female home range size as a guide we placed a $2 \mathrm{~km}^{2}$ grid over the study area for the occupancy framework. We considered each grid cell a 'site'. The camera locations were management based which resulted in some grids cells having multiple cameras and many grid cells having no cameras. Since we were interested in site-usage, and not abundance or density, identification of individual feral swine was not necessary, which reduced 


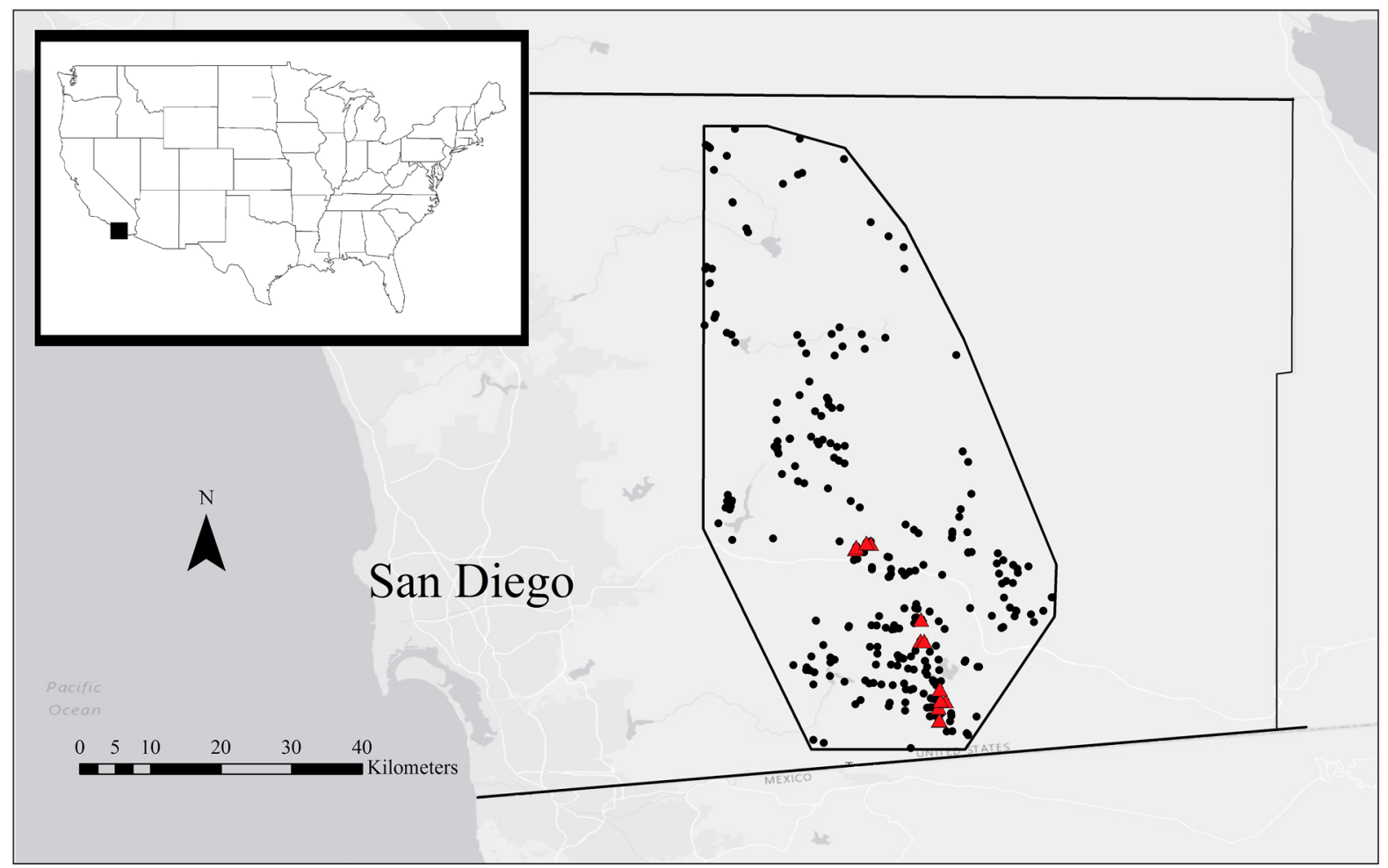

Fig. 2 Camera trap locations (black dots) for feral swine surveillance in San Diego County, California. Locations of feral swine removals are shown as red triangles

the time spent examining photos. Detections of feral swine in the area were generally low; therefore, we considered each week a single sampling occasion (any feral swine observed on a camera within 1 week was counted as a detection). We were interested in changes in feral swine site-usage across space and time and thus used months as a primary sampling period (the population is assumed to be open to changes in siteusage status among months) and weeks as the secondary sampling period as per dynamic occupancy-model terminology (MacKenzie et al. 2006). We used a simple binary value ' 1 ' indicating the species was detected during the sampling occasion and ' 0 ' indicating non-detection, regardless of the number of detections in a sampling period (Otis et al. 1978).

We analyzed the dynamic site-usage data using Robust Design Occupancy analysis implemented in program MARK (White and Burnham 1999). We used the parameterizations that models probability of siteusage for the first time step $\left(\Psi_{1}\right)$ and allows for modeling time-varying transition parameters on the local extinction rate ( $\varepsilon$-probability of a used site becoming unused) and local colonization ( $\gamma$-probability of an unused site becoming used). The site-usage probability for all remaining time steps can be calculated by site ' $i$ ' and time ' $t$ ' as follows:

$\psi_{i t}=\psi_{i t-1} *\left(1-\varepsilon_{i t-1}\right)+\left(1-\psi_{i t-1}\right) * \gamma_{i t-1}$

These models also allowed detection probability $(p)$ to vary by time and visit.

The time frame that each camera was active was not consistent across all cameras due to the accessibility of certain properties, logistical constraints of management actions, and the fact that some cameras were stolen. To account for the staggered nature and lack of consistent effort among all cameras we modeled detection as a function of the number of days cameras were active during each sampling period in each site (termed 'effort'). Additionally, some sites had more than one camera and therefore the number of camera nights accounts for the number of active cameras per site. 
Changes in distribution over time

We tested changes in site-usage across space and time. To test these effects we compared models with a linear time trend (by month, " $T$ ") and spatial movement (latitude and longitude, termed 'North' and 'East' for simplicity in displaying results) and an interaction between time and spatial effects for local extinction and colonization rates. We also tested whether the probability of local colonization was impacted by having a neighboring site being used to account for site-level movement of feral swine ( 0 - no queen's neighbors were used at time $t, 1$-at least one queen's neighbors site was used at time $t$ ).

Factors affecting distribution

First, we used the most parameterized models on initial site-usage ( $\psi_{\mathrm{i} 1}$ East and North, Eq. 2$)$ and the local extinction rate (East $\times$ Trend + North $\times$ Trend, Eq. 3) to determine which factors were important to the local colonization rate $\left(\gamma_{\text {it }}\right.$ including: East, North, Trend, East $\times$ Trend, North $\times$ Trend, and Neighbor effects, Eq. 4). Using the most parsimonious model on the colonization rate we compared models on the local extinction rate $\left(\varepsilon_{i t}\right)$. Using the most parsimonious models on the local extinction and colonization rates we compared factors on initial siteusage. We compared models using Akaike information criterion corrected for small sample size (AIC $c$, Burnham and Anderson 2002). We used a $\triangle \mathrm{AICc}$ of 2 as the cutoff to indicate substantial differences between models (Burnham and Anderson 2002).

$$
\begin{aligned}
\operatorname{logit}\left(\psi_{i 1}\right)= & \beta_{\psi 0}+\beta_{\psi 1} * \text { East }_{i}+\beta_{\psi 2} * \text { North }_{i} \\
\operatorname{logit}\left(\varepsilon_{i t}\right)= & \beta_{\varepsilon 0}+\beta_{\varepsilon 1} * \text { East }_{i}+\beta_{\varepsilon 2} * \text { North }_{i}+\beta_{\varepsilon 3} * T \\
& +\beta_{\varepsilon 4} * \text { East }_{i} * T+\beta_{\varepsilon 5} * \text { North }_{i} * T
\end{aligned}
$$

$$
\begin{aligned}
\log i t\left(\gamma_{i t}\right)= & \beta_{\gamma 0}+\beta_{\gamma 1} * \text { East }_{i}+\beta_{\gamma 2} * \text { North }_{i}+\beta_{\gamma 3} \\
& * T+\beta_{\gamma 4} * \text { East }_{i} * T+\beta_{\gamma 5} * \text { North }_{i} * T \\
& +\beta_{\gamma 6} * \text { Neighbor }_{i t}
\end{aligned}
$$

Effects of removals on site-usage rates

To examine the impact of feral swine removal efforts on site-usage rates we added the monthly removal totals by grid cell as a covariate on the probability of non-usage of sites to the top model. We used a likelihood-ratio test to compare the relative parsimony of the top model with and without the removal covariate.

Certainty in non-site-usage

To estimate detection probability accounting for the number of active cameras and the number of nights the cameras were active:

$P\left(\right.$ non-detection $_{i t} \mid$ usage $\left._{i t}\right)=\theta_{i t}=\left(1-p_{i t}\right)^{k_{i t}}$

$\operatorname{logit}\left(p_{i t}\right)=\beta_{0}+\beta_{1} * C_{i t}$

where $p_{i t}$ is the detection probability given the effort in site ' $i$ ' at time ' $t$ ' and $k_{i t}$ is the number of visits within a primary period (i.e., number of weeks in a month, 4 or 5). $\theta_{i t}$ is the probability of non-detection given a site is occupied. Detection probability $\left(p_{i t}\right)$ is modeled as a logit linear function of the number of camera nights $\left(\mathrm{C}_{\mathrm{it}}\right.$ - sum of the number of active nights for each camera in the site) in a given site ' $i$ ' at time ' $t$ '.

An additional objective of this study was to determine areas in our study that had lower certainty of not being used during time ' $t$ ' (referred to as absence) to highlight areas where increased management effort would be beneficial. We used site-usage information from our analyses and the sampling effort to determine the certainty of absence (MacKenzie et al. 2006).

$$
\begin{aligned}
& P\left(\text { absence }_{i t} \mid \text { no detections }_{i t}\right) \\
& =\frac{\left(1-\Psi_{i t}\right)}{\Psi_{i t} *\left(1-p_{i t}\right)^{k_{i t}}+\left(1-\Psi_{i t}\right)}
\end{aligned}
$$

where $\Psi_{i t}$ is the probability of site-usage at site ' $i$ ' at the last time step, and $p_{i t}$ and $k_{i t}$ are defined as above.

\section{Certainty in elimination}

Next we demonstrated how our approach could be used to predict the certainty of site-level absence across space in our study area. Because elimination certainty is only relevant when a species is not 
detected for some period of time (not the case in our data), we simulated a camera sampling design similar to our study area during the last month of observations but with negative surveillance data during the 5 months following the end of our study. Based on the monthly negative surveillance data during the last 5 months, we then projected the certainty in site-level absence in the study area given no feral swine were detected and using the detection probability from times when feral swine were present. We used the top model determined from our analysis to predict elimination certainty.

\section{Costs}

We recorded the hours worked for both camera surveillance and removal activities for the duration of the study. We also recorded the numbers of cameras and traps used in our study in order to give calculate average costs for similar work.

\section{Results}

During the study there were 933 total photographs of feral swine taken (Fig. 3), which resulted in 351 sampling events with detections (pooled by week and grid cell). The total amount of effort employed for the camera trapping was 54,420 camera nights (sum of the total number of nights that all cameras were active). There were 48 feral swine removed as a result of trapping or ground shooting (locations shown in Fig. 2).

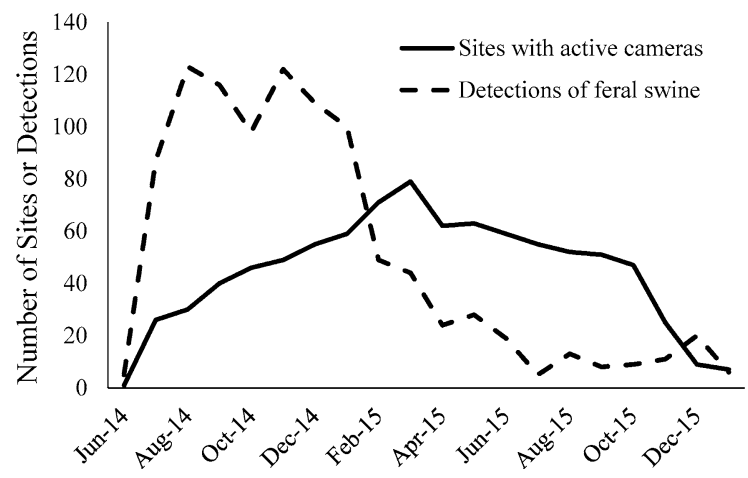

Fig. 3 Number of sites with active cameras over time (solid line) and the number of detections of feral swine across time (dashed line)
The most parsimonious model on colonization rates included both spatial direction parameters (East and North) as well as a neighbor effect (Tables 1 and S1). Colonization rates were higher in the south than in the north and were higher in the west than the east (Tables 1 and S1). Colonization rates were 4.6 times higher (95\% CI 4.1-5.1 times higher) for sites that did not have a neighboring site that was used compared to those that did have a neighboring site that was used. The most parsimonious model on the local extinction rate included an east effect added with a time trend (Tables 1 and S1). Local extinction rates were higher in the west than in the east (Table 1), and local extinction rates increased across months from 0.16 (SE 0.05) at the start of the study to 0.63 (SE 0.12) at the end of the study (Table 1).

Site-usage probabilities were higher in the south than the north, generally. Overall site-usage in San Diego County was fairly low at the beginning of our study $(\widehat{\Psi}=0.13$, SE 0.13$)$ and was reduced by $82 \%$ by the end of the study ( $\widehat{\Psi}=0.024$, SE 0.007; Fig. 4; variance of estimates by month are shown in Appendix Fig S2). We examined the impact of removal efforts on local extinction rates by adding removal to the top model as a covariate on $\varepsilon$. The likelihood ratio test did not support addition of the removal effect as a factor influencing overall occupancy ( $p$ value for the L-R test 0.51). However, the parameter estimate for the removal effect did suggest that removal events

Table 1 Parameter estimates and standard error for the top model on dynamic occupancy of feral swine in San Diego County, California

\begin{tabular}{lrr}
\hline Parameter & Estimate & SE \\
\hline$\Psi_{1}$ Intercept & 16.35 & 9.42 \\
$\Psi_{1}$ North & -0.90 & 0.54 \\
$\Psi_{1}$ East & -0.67 & 0.32 \\
$\varepsilon$ Intercept & 1.17 & 0.91 \\
$\varepsilon$ East & -0.29 & 0.08 \\
$\varepsilon$ Trend & 0.12 & 0.04 \\
$\gamma$ Intercept & -1.97 & 0.98 \\
$\gamma$ Neighbor & 1.58 & 0.51 \\
$\gamma$ East & -0.14 & 0.06 \\
$\gamma$ North & -0.07 & 0.04 \\
p Intercept & -0.85 & 0.14 \\
$\mathrm{p}$ Effort & 0.03 & 0.01 \\
\hline
\end{tabular}


Fall 2014

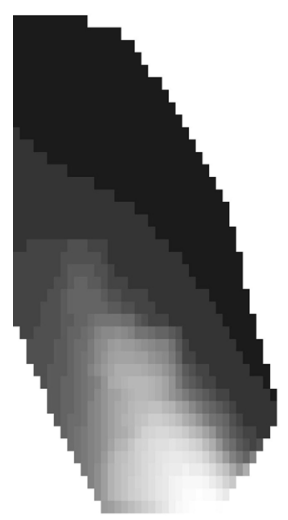

Summer 2015

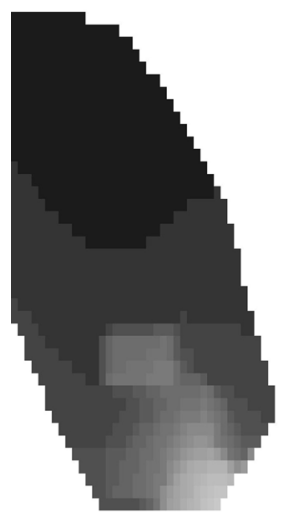

Winter 2014

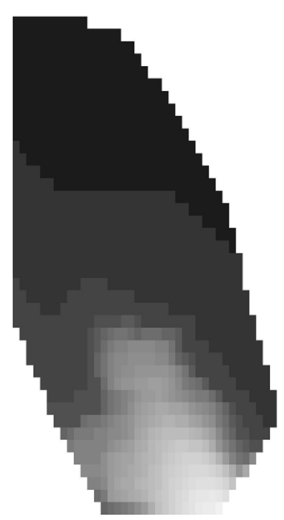

Fall 2015

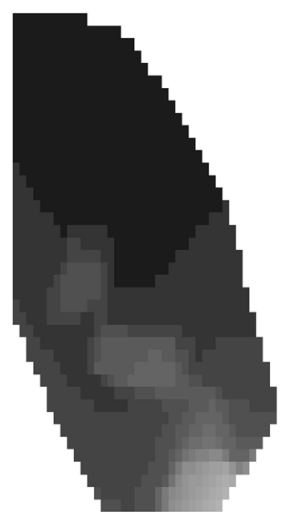

Spring 2015

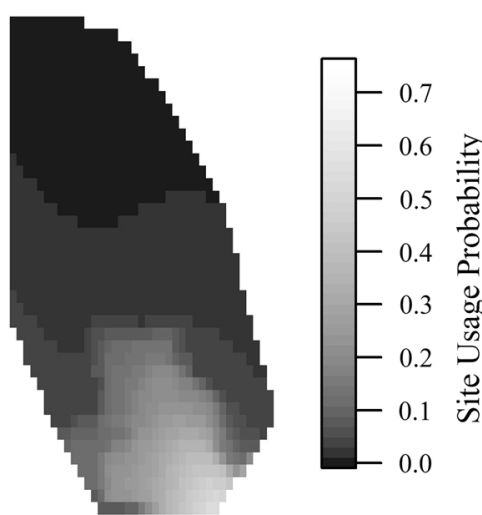

Winter 2015

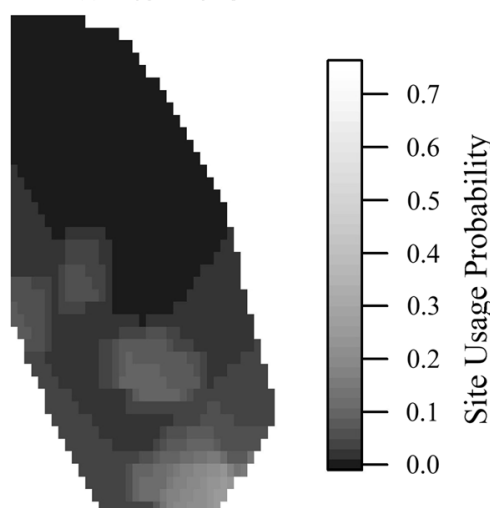

Fig. 4 Site-usage probability of feral swine by season in San Diego County, California, lighter shades indicate higher site-usage probabilities

increased local extinction rates (i.e. one additional removal results in an increase of 0.03 extinction rate SE 0.04), resulting in a decline in overall site-usage.

We highlighted spatial areas that needed more surveillance coverage by calculating the probability of not detecting feral swine if the site was used throughout the entire study (Fig. 5). There were larger areas with gaps in coverage in the north than in the south. We estimated the certainty of site-level absence during the last month of the study to highlight areas where increased surveillance should be focused and removal efforts should be concentrated (Fig. 6). Areas in the south and near previous detections had lower certainties of absence. The northern sites, despite having lower coverage, had higher certainties of not being used based on the dynamics of site-usage that we observed.

As would be expected, our approach shows increased certainty in elimination as more negative
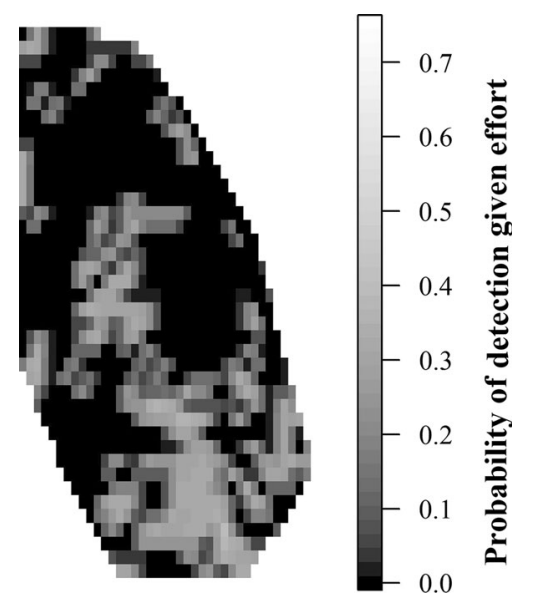

Fig. 5 Variation in effort across the study area. Probability of detecting feral swine if the site was used based on the number of active cameras by grid cell in San Diego County, California June 2014-January 2016 


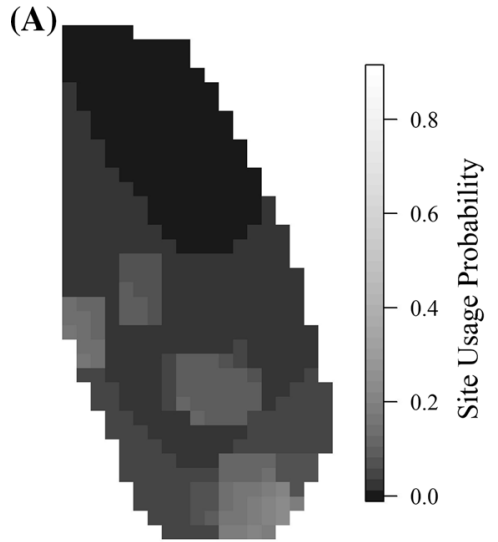

Fig. 6 a Probability of feral swine site-usage at the last time step in our study (January 2016) in San Diego County, CA. b Probability of detecting feral swine based on the number of active cameras per site at the last time step in our study (January 2016). c Probability of site-level absence of feral swine given

surveillance data were collected (results for 1, 3, and 5 months out are shown in Fig. 7), being lowest in sites with no surveillance effort. However, some sites with no surveillance effort show non-zero certainties because our model accounts for neighbor effects (i.e., sites that are adjacent to sites with high rates of negative surveillance are more likely to not have pigs). In our example, after 5 months of negative surveillance in the entire study area, the lowest site-level
(C)

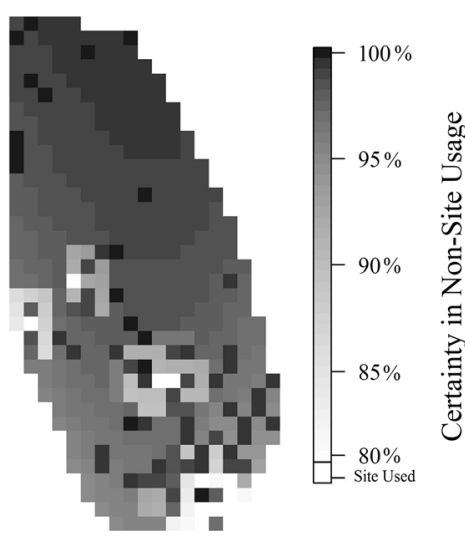

they were not detected and given the sampling effort involved. In areas where no surveillance was conducted the probability of site-level absence is simply 1-probability of site-usage. White sites indicate areas where there were detections of feral swine in the last time step

certainty of absence (where no surveillance was conducted) was $94.3 \%$, demonstrating that 5 months may be long enough to assume maximum control has been effective and newly observed pigs are likely to be reinvades.

We recorded a total of $9500 \mathrm{~h}$ of labor for the camera surveillance portion of the study. This includes time spent visiting sites, baiting cameras, setting up cameras, monitoring cameras, and reviewing photos

\section{Average site-usage probability}

Jun 2014 - Jan 2016

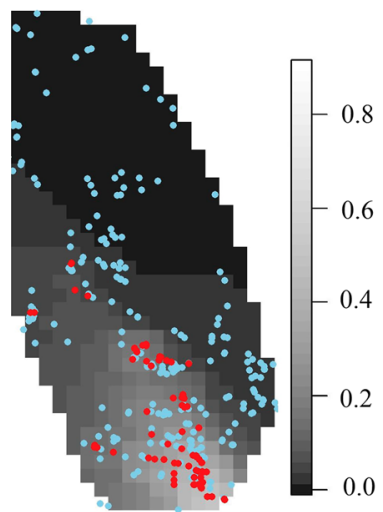

Feb 2016

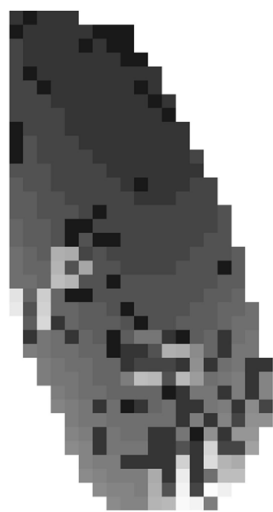

Fig. 7 The left plot is an average of the site-usage probability during the entire study (Jun 2014-Jan 2016). The camera locations are shown with no detections in blue and with feral swine detections in red. The next three plots are the certainty of

\section{Predicted certainty of site-level absence}

Apr 2016

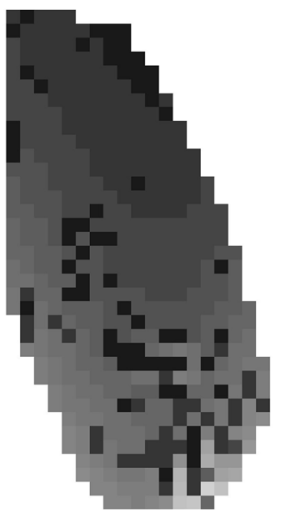

Jun 2016

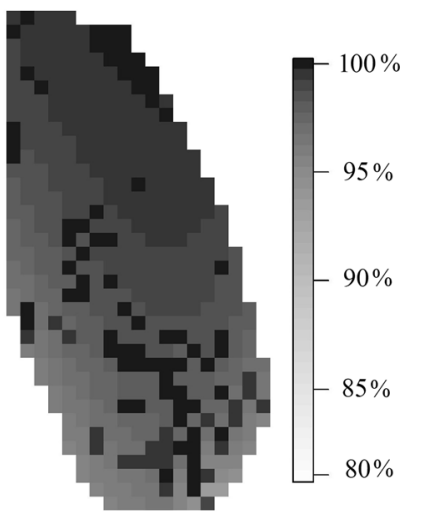

site-level absence by space and time accounting for surveillance effort when no feral swine have been detected for February, April, and June 2016 despite ongoing surveillance 
from cameras for wild pig activity. We recorded $1702 \mathrm{~h}$ of labor for removal work. This work includes setting up traps, baiting traps, checking traps, euthanizing wild pigs caught in traps, and hunting for wild pigs. We used a total of 285 cameras and 12 traps. The upfront costs per camera were $\$ 220$ and the average labor per camera during the study was $33 \mathrm{~h}$. The upfront costs per trap were $\$ 635$ and the average labor per trap was $141 \mathrm{~h}$ per trap.

\section{Discussion}

For programs in which the goal is to eliminate an invasive species from a target area when there is risk of reinvasion, important questions are: "where are we most likely to find the species?", and "what is the probability it is absent at specific sites given our surveillance effort?" Answers to these questions can improve efficiency of resource distribution by providing guidance for how to optimize resource allocation in space and time. Using Bayes theorem we calculated the conditional probability of site-level absence within 1 month given effort, detection, and site-usage (MacKenzie et al. 2006). We applied this method to our management-based surveillance design to demonstrate how conditional probability of site-level absence can be estimated temporally using commonly collected management data. We determined that in areas with a realistic number of cameras $(<5)$ the certainty of site-level absence can be very high ( $\sim 95 \%$ ) once no detections occur for 5 months. Additionally, in areas where there were no cameras, we were more confident that there was site-level absence in the northern sites compared to the southern sites over the course of our study. Therefore, even though the sampling intensity in the north is much lower than in the south (fewer grid cells with cameras), it would be more beneficial to place new cameras in sites in the south to help address the greater uncertainty in the elimination in the south than in the north.

There are several studies that give guidance as to the optimal study design for occupancy analyses depending on the species (e.g., MacKenzie and Royle 2005; Shannon et al. 2014). It has been shown that effort allocation (e.g., the number of sites monitored and the number of visits to each site) should be modified to fit the particular scenario and questions being asked. Specifically, the estimated site usage and detection rates influence how sampling should be allocated. For common species with high detection, the number of visits should be increased, while for species with low site usage and low detection, both the number of sites sampled and the number of visits should be increased (MacKenzie and Royle 2005; Shannon et al. 2014). For situations similar to our study (low site usage and moderate detection), Shannon et al. (2014) recommend having a moderate number of sites sampled but increase the number of sampling occasions. Based on our sampling approach (examining weekly detections within a month) we have a fixed number of repeated samples possible by primary period (month). MacKenzie and Royle (2005) suggest that based on the number of visits (weeks in our model) for the site usage we observed (0.10), and the detection rate we observed (0.30), we should have 5 visits per primary period, which is encouraging as 4-5 weeks per month was the number of repeated samples.

An important consideration for our and any elimination program is that surveillance must be ongoing throughout the maximum control and EDRR phases. For example, it is inappropriate to assume that predictions of absence certainty that are based on data collected during the final stages of maximum control would apply in the long-term. Rather our framework would need to be refit iteratively with the most current surveillance data because animals are mobile such that reinvasion risk at particular sites will change and absence cannot be assumed as a permanent state. However, for elimination programs, being able to assess the certainty with which an area is devoid of a species is especially important because there are two phases of management: maximum control, where surveillance and control occur throughout the managed area, and EDRR where surveillance and response are focused on areas with high-reinvasion risk (Fig. 1b). During the maximum control phase, the goal of surveillance is to highlight areas where removal work should be concentrated. Once the population reaches low-density, control becomes less efficient and there is uncertainty when to stop searching for individuals throughout the study area. Our approach can be used to determine when this switch from area-wide surveillance to surveillance focused on reinvasions should be made. For any particular system, a threshold of elimination certainty in the managed area (for example 95\%) can be set for 
determining the transition from phase 1 to phase 2 surveillance. In our system, it appeared that a $95 \%$ threshold could be achieved after 5 months of negative surveillance data, suggesting that surveillance to declare elimination need not be too long if in fact no individuals are observed. In addition, cost data could be included into the decision for choosing which threshold of certainty is appropriate.

There are several studies that have examined the probability of detecting differences in certainty in absence as it relates to survey effort, detection, and occupancy probabilities (Ellis et al. 2014; GuilleraArroita and Lahoz-Monfort 2012). Increasing the detection $(p)$ relates to greater power to detect changes in site-usage status compared to increasing the number of sites visited or the number of visits in some cases (Ellis et al. 2014; Guillera-Arroita and Lahoz-Monfort 2012). In our situation, a way to increase detection probability was to increase the number of cameras per grid cell. Certainty in elimination was more strongly influenced by an increase in detection than extending the time spent surveillance (Fig. 7).

Estimating a spatial surface of absence certainty can be particularly useful in systems where reinvasion risk is substantial. When analyses show high certainty of absence over much of the study area, the program can shift resources to focus on EDRR (Rabaglia et al. 2008; Westbrooks 2004) strategies rather than studywide control campaigns. Passive camera traps are a commonly used, low-cost method to surveillance wildlife populations, particularly when the only response of interest is presence/absence (no individual identification is needed). Our results show the upfront costs and labor required per camera are considerably less than is needed for trapping work. This type of work could be used to optimize the camera design needed to conduct initial removal and reinvasion control most efficiently.

Intensive feral swine control has been conducted in San Diego County, California by managers since 2014. Correspondingly, overall site usage of feral swine has declined from June 2014 to January 2016. Although our results indicate that removal events were related to increases in local extinction rates (and thus declines in overall site usage), the removal events were not as strongly tied to extinction rates as other factors. One likely reason for the weak correspondence is that the number of feral swine removed per event was small ( $\bar{x}=4.5$ individuals $)$ and thus individual removals may not have corresponded with a change in site-usage status (e.g., if one individual remained at a site after a removal event the site usage will not have changed). Additionally, other control actions, that were unaccounted for, may have been conducted in the area (e.g., ground shooting by other agencies, hunters, or land owners) and impacted site usage. This analytical framework is focused on evaluating patterns of site usage, to examine the efficacy of removal methods a different analysis focused on impact of removals should be used (e.g., a method that examines population size change and not site usage). However, when densities are low the results of an analysis targeted at estimating the effectiveness of removal actions may be too variable to produce strong relationships.

One of the objectives of this study was to determine the spatial distribution of feral swine on the landscape and to determine if the site-usage patterns were changing over time. By examining directional changes in site usage through time, we determined that the range of feral swine has contracted to the southerncentral portion of the study site during the 20-month surveillance time (Fig. 4). This decline in the study area could be due to a range-wide decline or to movement south and out of the study area. Either option is beneficial for our study area, however, if feral swine are simply moving out of the study area into other areas this may suggest that immigration back to our study area is possible. Extending the surveillance to areas surrounding the study area particularly in the south and implementing an EDRR program in that area could help maintain elimination status in the rest of the target area (Adams et al. 2014; Westbrooks 2004). Additionally, it will be important to continually update the model selection process to identify current factors that influence the dynamic occupancy process as new information becomes available and not assume our results (e.g., a southerly movement of occupied sites) will continue, as this system is dynamic and subject to change as outside influences change.

Evaluation of changes in feral swine populations in this study was possible due to surveillance by camera traps. Camera traps are commonly used by wildlife managers and land owners to monitor wildlife on their properties for guiding controls (O'Connell et al. 2010). It would be valuable to use this regularly collected data to answer questions about population distribution changes, even when the placement of cameras is not 
set up following a statistical study design. Our results demonstrated the ability of management-based camera-trap data to monitor and quantify changes in site usage across time and test the impacts of management actions. The ability to make inference to sites not sampled is dependent on the representativeness of the sites that were sampled to those that were not. In this situation, sites that were sampled are more likely to be have feral swine presence than sites that were not sampled as they are targeted by managers for that purpose. Therefore, the site-usage estimates may be biased high in the non-sampled sites compared to those that were sampled. For management applications this direction of bias may be preferable as minimizing the false negative error (claiming feral swine are absent when they are not) is a larger problem than false positive error rates (claiming presence when they are absent). Claiming feral swine are absent when they are not is a larger issue because it could lead to a decline in removal and management efforts in the area which could allow the population to rebound and expand, causing more damage than the expense of continued surveillance if the error were the other way. If the bias were the other way and camera placement was less likely to detect wild pigs than we would be underestimating the occupancy of wild pigs which would be a considerable problem. Previous work on wild pigs has shown that baiting usually attracts pigs within a $\sim 1.7 \mathrm{~km}$ radius (Davis et al. 2017) and up to $10 \mathrm{~km}$ (N. Snow unpublished data). Therefore, it is likely that pigs within the $2 \mathrm{~km} \times 2 \mathrm{~km}$ sites would be detected if present. If concerns about this bias exist it would be worthwhile to place cameras randomly in some areas in order to test this assumption. However, we do not believe that is a problem in our area for reasons we have stated.

It would also be beneficial to use study design and occupancy theory to provide guidance on future surveillance and provide interpretations for surveillance results. The camera placements in our study allowed us to monitor $\sim 29 \%$ of the sites (grid cells). We used probability theory to determine the conditional probability of not-detecting feral swine given they were present (Fig. 5) to demonstrate areas that have poor coverage (MacKenzie et al. 2006, 2009). However, optimal camera allocation is dependent not only on sampling effort but also on the probability of site usage (MacKenzie et al. 2006). Here we examined site usage as it relates to spatial factors (latitude, longitude, and neighbor effects). Our study area was fairly homogeneous and we had relatively few areas with detections and thus we did not examine habitat effects directly. However, a potential avenue for similar studies to increase the precision around estimates is to include habitat covariates that relate to site usage. Currently, we are able to use the probability of site-level absence at the last time step to provide recommendations as to where future surveillance and removal efforts should be concentrated. Such guidance allows for more efficient use of limited surveillance resources (Lavoie et al. 2007).

\section{Conclusions}

Passive camera trapping can be used to assess population distribution changes of mammals, like feral swine. Analyzing camera data using site-usage analysis which accounts for sampling design, allows for: (1) determining changes in species distribution over space and time, (2) assessing effects of management in low-density populations, and (3) prioritizing areas for increased surveillance or targeted removal efforts. If the primary objective is to assess changes across time than increasing the detection probability of the species of interest will have the greatest impact, and we found that can be achieved by increasing the number of cameras per grid cell. If managers are interested in certainty of site-level absence, increased effort should be concentrated in areas with higher probability of site-usage. By analyzing detection data in an occupancy modeling framework, gaps in the surveillance coverage can be highlighted and adaptive surveillance designs that improve surveillance effectiveness over space and time can be achieved. Our approach can be especially useful for deciding when elimination has been achieved, such that resources may be focused on prevention, surveillance, and response to reinvasions.

Acknowledgements Funding was provided by the U.S. Department of Agriculture Animal and Plant Health Inspection Service Wildlife Services division. Thanks to the Wildlife Services personal: Joe Albee, and Chris Freitas, who collected and organized the data for this project. Any use of trade, firm, or product names is for descriptive purposes only and does not imply endorsement by the U.S. government.

Data archiving Data associated with this manuscript will be archived in Dryad Data Repository. 


\section{References}

Adams A, van Heezik Y, Dickinson K et al (2014) Identifying eradication units in an invasive mammalian pest species. Biol Invasions 16:1481-1496

Allendorf FW, Lundquist LL (2003) Introduction: population biology, evolution, and control of invasive species. Conserv Biol 17:24-30

Anderson DP, Ramsey DSL, Nugent G et al (2013) A novel approach to assess the probability of disease eradication from a wild-animal reservoir host. Epidemiol Infect 141:1509-1521

Baber DW, Coblentz BE (1986) Density, home range, habitat use, and reproduction in feral pigs on Santa Catalina Island. J Mammal 67:512-525

Bevins SN, Pedersen K, Lutman MW et al (2014) Consequences associated with the recent range expansion of nonnative feral swine. Bioscience 64:291-299

Bomford M, O'Brien P (1995) Eradication or control for vertebrate pests? Wildl Soc Bull 1973-2006(23):249-255

Burnham KP, Anderson DR (2002) Model selection and multimodel inference-a practical information-theoretic approach. Springer, New York

Crowl TA, Crist TO, Parmenter RR et al (2008) The spread of invasive species and infectious disease as drivers of ecosystem change. Front Ecol Environ 6:238-246

Davis AJ, Hooten MB, Miller RS et al (2016) Inferring invasive species abundance using removal data from management actions. Ecol Appl 26:2339-2346

Davis AJ, Leland B, Bodenchuk M et al (2017) Estimating population density for disease risk assessment: the importance of understanding the area of influence of traps using wild pigs as an example. Prevent Vet Med 141:33-37

Dowdle WR (1998) The principles of disease elimination and eradication. Bull World Health Organ 76:22

Edge K, Crouchley D, McMurtrie P et al (2011) Eradicating stoats (Mustela erminea) and red deer (Cervus elaphus) off islands in Fiordland. In: Veitch CRC, Clout MN, Towns DR (eds) Island invasives. Eradication and Management, NewYork

Ellis MM, Ivan JS, Schwartz MK (2014) Spatially explicit power analyses for occupancy-based monitoring of wolverine in the U.S.A, Rocky Mountains. Conserv Biol 28:52-62

Engeman RM, Massei G, Sage M et al (2013) Monitoring wild pig populations: a review of methods. Environ Sci Pollut Res 20:8077-8091

Guillera-Arroita G, Lahoz-Monfort JJ (2012) Designing studies to detect differences in species occupancy: power analysis under imperfect detection. Methods Ecol Evol 3:860-869

Hoehne VN (1994) Wild pigs in Santa Cruz County. Fremontia 22:18-19

Kendall WL, Hines JE, Nichols JD et al (2013) Relaxing the closure assumption in occupancy models: staggered arrival and departure times. Ecology 94:610-617

Lavoie C, Donlan CJ, Campbell K et al (2007) Geographic tools for eradication programs of insular non-native mammals. Biol Invasions 9:139-148
Lyons JE, Runge MC, Laskowski HP et al (2008) Monitoring in the context of structured decision-making and adaptive management. J Wildl Manag 72:1683-1692

MacKenzie DI (2005) Was it there? Dealing with imperfect detection for species presence/absence data. Aust N Z J Stat 47:65-74

MacKenzie DI, Royle JA (2005) Designing occupancy studies: general advice and allocating survey effort. J Appl Ecol 42:1105-1114

MacKenzie DI, Nichols JD, Sutton N et al (2005) Improving inferences in population studies of rare species that are detected imperfectly. Ecology 86:1101-1113

MacKenzie DI, Nichols JD, Royle JA et al (2006) Occupancy estimation and modelling-inferring patterns and dynamics of species occurrence. Academic Press, London

MacKenzie DI, Nichols JD, Seamans ME et al (2009) Modeling species occurrence dynamics with multiple states and imperfect detection. Ecology 90:823-835

Mayer JJ, Brisbin IL (2008) Wild pigs in the United States: their history, comparitive morphology, and current status. University of Georgia Press, Athens

Myers JH, Simberloff D, Kuris AM et al (2000) Eradication revisited: dealing with exotic species. Trends Ecol Evol 15:316-320

O'Connell AF, Nichols JD, Karanth KU (2010) Camera traps in animal ecology: methods and analyses. Springer, New York

Otis DL, Burnham KP, White GC et al (1978) Statistical inference from capture data on closed animal populations. Wildl Monogr 62:3-135

Parkes JP, Nugent G, Forsyth DM et al (2017) Past, present and two potential futures for managing New Zealand's mammalian pests. N Z J Ecol 41:151-161

Rabaglia R, Duerr D, Acciavatti R et al (2008) Early detection and rapid response for non-native bark and ambrosia beetles. US Department of Agriculture Forest Service, Forest Health Protection, New York

Ramsey DSL, Parkes JP, Morrison SA (2009) Quantifying eradication success: the removal of feral pigs from Santa Cruz Island, California. Conserv Biol 23:449-459

Regan TJ, McCarthy MA, Baxter PWJ et al (2006) Optimal eradication: when to stop looking for an invasive plant. Ecol Lett 9:759-766

Robertson PA, Adriaens T, Lambin X et al (2016) The largescale removal of mammalian invasive alien species in Northern Europe. Pest Manag Sci 73:273-279

Roemer GW, Donlan CJ, Courchamp F (2002) Golden eagles, feral pigs, and insular carnivores: how exotic species turn native predators into prey. Proc Natl Acad Sci 99:791-796

Rout TM, Kirkwood R, Sutherland DR et al (2014) When to declare successful eradication of an invasive predator? Anim Conserv 17:125-132

Saunders G, McLeod S (1999) Predicting home range size from the body mass or population densities of feral pigs, Sus scrofa (Artiodactyla: Suidae). Aust J Ecol 24:538-543

Seber GAF (1982) The estimation of animal abundance and related parameters, 2nd edn. Charles and Griffin and Company Limited, London

Seward NW, Ver Cauteren KC, Witmer GW et al (2004) Feral swine impacts on agriculture and the environment. Sheep Goat Res J 12:34-40 
Shannon G, Lewis JS, Gerber BD (2014) Recommended survey designs for occupancy modelling using motion-activated cameras: insights from empirical wildlife data. PeerJ 2:e532

Simpson A, Jarnevich C, Madsen J et al (2009) Invasive species information networks: collaboration at multiple scales for prevention, early detection, and rapid response to invasive alien species. Biodiversity 10:5-13

Thorn M, Scott DM, Green M et al (2009) Estimating brown hyaena occupancy using baited camera traps. South Afr J Wildl Res 39:1-10
Tobler MW, Zúñiga Hartley A, Carrillo-Percastegui SE et al (2015) Spatiotemporal hierarchical modelling of species richness and occupancy using camera trap data. J Appl Ecol 52:413-421

Westbrooks RG (2004) New approaches for early detection and rapid response to invasive plants in the United States. Weed Technol 18:1468-1471

White GC, Burnham KP (1999) Program MARK: survival estimation from populations of marked animals. Bird Study 46:S120-S139 

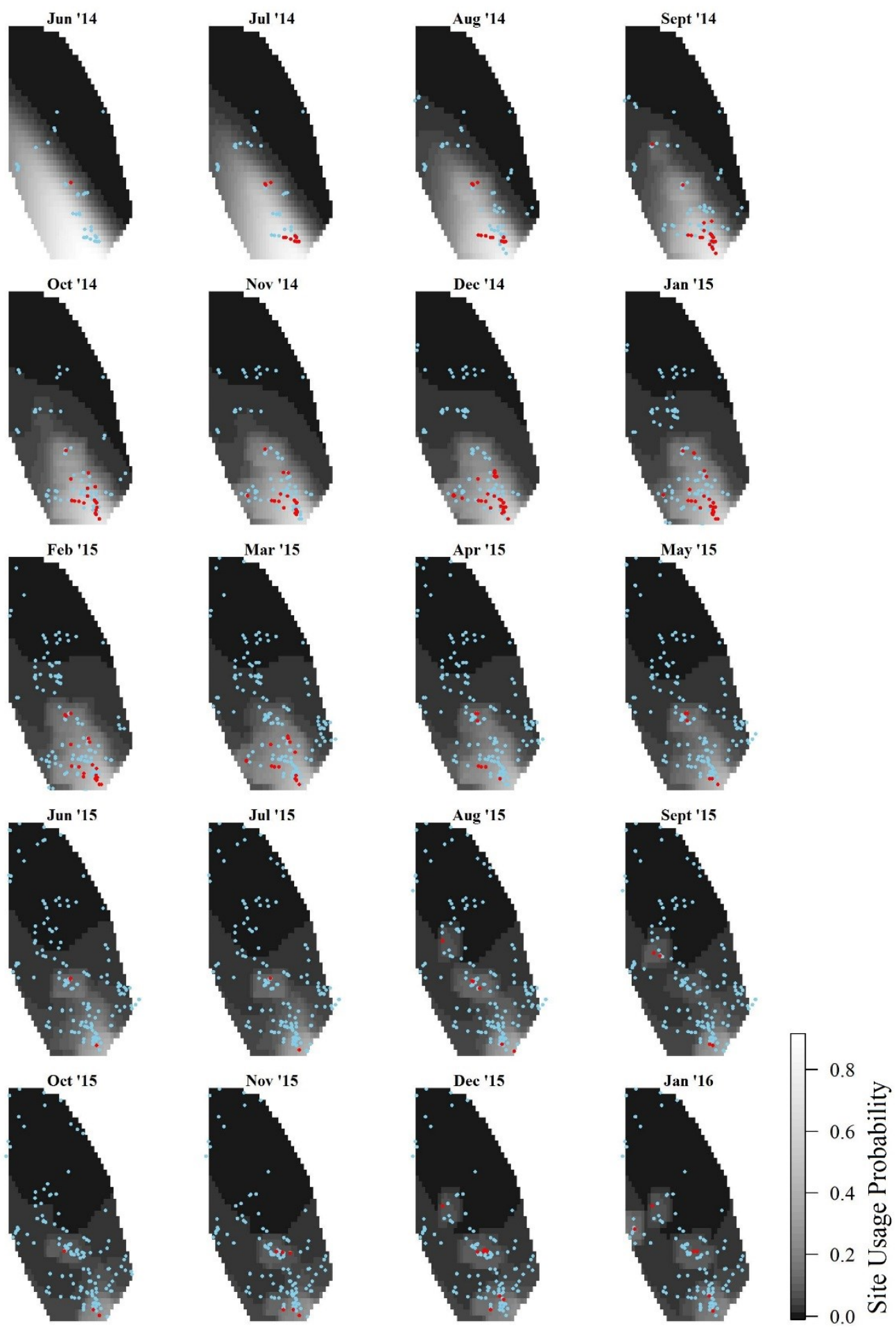

Fig S1. Site usage probability of feral swine in San Diego County, CA from June 2014 to Jan 2016 shown by month with active cameras shown both with detections (red) and without detections (blue). 

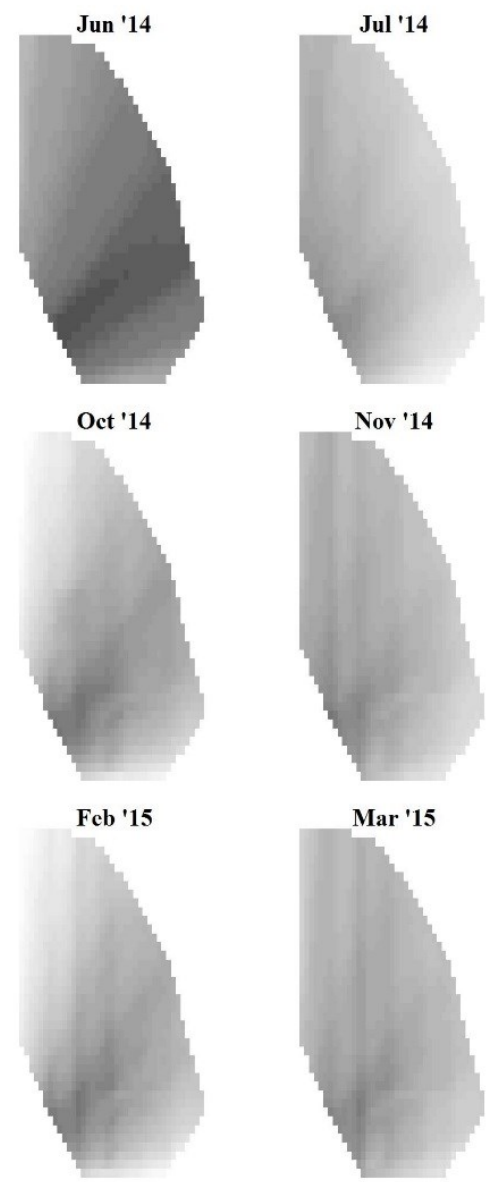

Jun '15

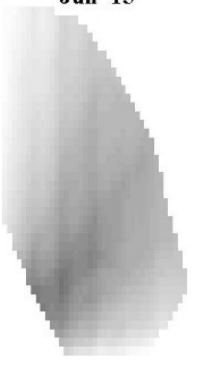

Oct '15

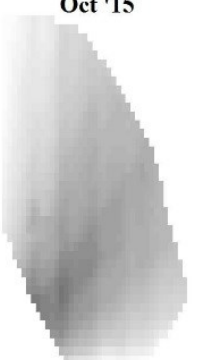

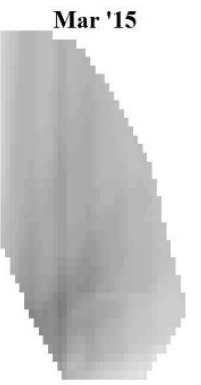
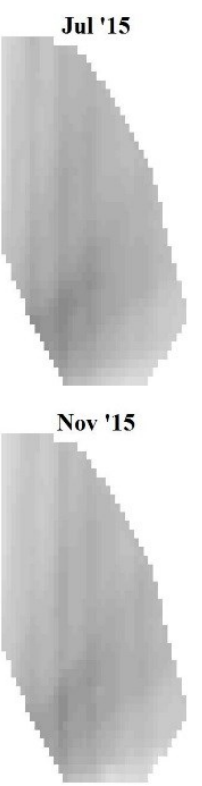

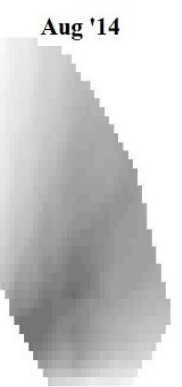

$$
\text { Dec '14 }
$$
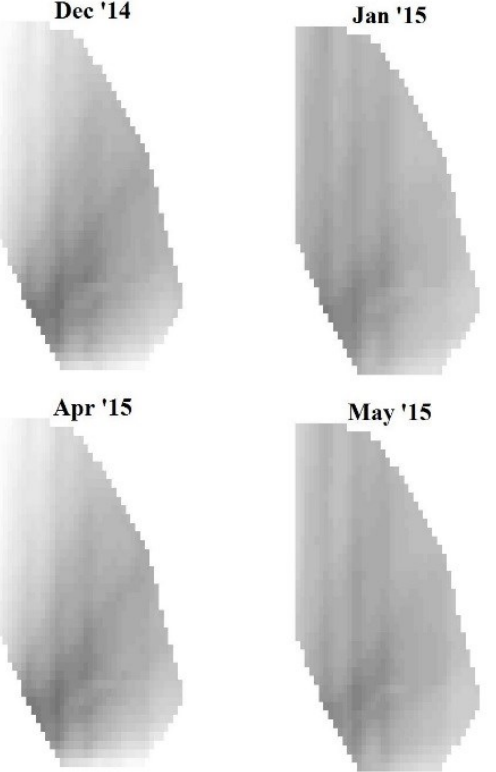

$$
\text { Aug '15 }
$$
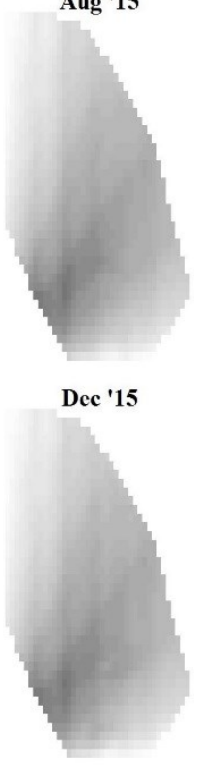

Fig S2. Variance of site usage probability of feral swine in San Diego County, CA from June 2014 to Jan 2016. 
Table S1. Model results for dynamic occupancy of feral swine in San Diego County, California from July 2014 to January 2016. Initial colonization $\left(\Psi_{1}\right)$, local extinction $(\varepsilon)$, and colonization $(\gamma)$ are modeled with respect to directional elements and linear time trend (T). Models are ranked using Akaike information criterion corrected for small sample size (AICc). The difference $(\Delta)$ from the model with the lowest AICc value are shown (lowest AICc $=1448.02$ ). The number of parameters $(\mathrm{k})$ and deviance are shown.

\begin{tabular}{|c|c|c|c|c|}
\hline Model & $\triangle \mathrm{AICc}$ & $\mathrm{AIC}_{\mathrm{c}} w$ & $\mathrm{k}$ & Deviance \\
\hline$\Psi 1($ East+North $) \varepsilon($ East $+\mathrm{T}) \gamma($ Neib+East+North)p(effort $)$ & 0.00 & 0.28 & 12 & 1423.80 \\
\hline$\Psi 1($ East + North $) \gamma($ Neib + East + North $) \varepsilon($ East*T $) p($ effort $)$ & 1.17 & 0.16 & 13 & 1422.94 \\
\hline$\Psi 1($ East + North $) \varepsilon($ East + North $+\mathrm{T}) \gamma($ Neib + East + North $) p($ effort $)$ & 1.43 & 0.14 & 13 & 1423.19 \\
\hline$\Psi 1($ East + North $) \varepsilon($ East $+\mathrm{T}+$ Removal $) \gamma($ Neib + East + North $) p($ effort $)$ & 1.61 & 0.12 & 13 & 1423.37 \\
\hline$\Psi 1($ East + North $) \gamma($ Neib+East + North $) \varepsilon($ East*T+North $) p($ effort $)$ & 2.30 & 0.09 & 14 & 1422.03 \\
\hline$\Psi 1($ East + North $) \varepsilon($ East*T+North*T $) \gamma($ Neib + East + North $) p($ effort $)$ & 3.80 & 0.04 & 15 & 1421.49 \\
\hline$\Psi 1($ East + North $) \varepsilon($ East*T+North*T) $\gamma($ Neib + East $) p($ effort $)$ & 4.10 & 0.04 & 14 & 1423.82 \\
\hline$\Psi 1($ East + North $) \varepsilon($ East $* \mathrm{~T}+$ North $* \mathrm{~T}) \gamma($ Neib $) \mathrm{p}($ effort $)$ & 4.26 & 0.03 & 13 & 1426.02 \\
\hline$\Psi 1($ East + North $) \varepsilon($ East*T+North $* \mathrm{~T}) \gamma($ Neib + East $+\mathrm{T}+$ North $) p($ effort $)$ & 5.58 & 0.02 & 16 & 1421.22 \\
\hline$\Psi 1($ East + North $) \varepsilon($ East*T+North*T $) \gamma($ Neib $+\mathrm{T}) \mathrm{p}($ effort $)$ & 5.92 & 0.01 & 14 & 1425.65 \\
\hline$\Psi 1($ East + North $) \varepsilon($ East $* \mathrm{~T}+$ North $* \mathrm{~T}) \gamma($ East $+\mathrm{T}+\mathrm{Neib}) \mathrm{p}($ effort $)$ & 5.94 & 0.01 & 15 & 1423.62 \\
\hline$\Psi 1($ East + North $) \varepsilon($ East*T+North*T $) \gamma($ Neib + North $) p($ effort $)$ & 6.19 & 0.01 & 14 & 1425.92 \\
\hline$\Psi 1($ East + North $) \varepsilon($ North $+\mathrm{T}) \gamma($ Neib+East + North $) p($ effort $)$ & 6.27 & 0.01 & 12 & 1430.07 \\
\hline$\Psi 1($ East + North $) \varepsilon($ East*T+North $* \mathrm{~T}) \gamma($ Neib + North $* \mathrm{~T}+$ East $) \mathrm{p}(\mathrm{effort})$ & 7.57 & 0.01 & 17 & 1421.16 \\
\hline$\Psi 1($ East + North $) \varepsilon\left(\right.$ East $^{*} \mathrm{~T}+$ North $\left.* \mathrm{~T}\right) \gamma($ Neib + East $* \mathrm{~T}+$ North $) \mathrm{p}(\mathrm{effort})$ & 7.58 & 0.01 & 17 & 1421.18 \\
\hline
\end{tabular}




\begin{tabular}{|c|c|c|c|c|}
\hline$\Psi 1($ East + North $) \varepsilon($ East*T+North*T $) \gamma($ Neib + North $+\mathrm{T}) \mathrm{p}($ effort $)$ & 7.82 & 0.01 & 15 & 1425.50 \\
\hline$\Psi 1($ East+North $) \varepsilon($ East*T+North*T) $\gamma($ Neib+East*T)p(effort $)$ & 7.94 & 0.01 & 16 & 1423.58 \\
\hline$\Psi 1($ East + North $) \varepsilon($ East*T+North*T $) \gamma($ Neib+East*T+North*T)p(effort) & 9.61 & 0.00 & 18 & 1421.16 \\
\hline$\Psi 1($ East + North $) \varepsilon($ East $* \mathrm{~T}+$ North $* \mathrm{~T}) \gamma($ Neib + North*T)p(effort $)$ & 9.86 & 0.00 & 16 & 1425.50 \\
\hline$\Psi 1($ North $) \varepsilon($ East+North $) \gamma($ Neib+East+North $) p($ effort $)$ & 9.98 & 0.00 & 11 & 1435.82 \\
\hline$\Psi 1(.) \varepsilon($ East+North $) \gamma($ Neib+East+North $) p($ effort $)$ & 10.96 & 0.00 & 10 & 1438.83 \\
\hline$\Psi 1($ East $) \varepsilon($ East + North $) \gamma($ Neib+East+North $) p($ effort $)$ & 11.52 & 0.00 & 11 & 1437.35 \\
\hline$\Psi 1($ East + North $) \varepsilon($ East*T+North*T $) \gamma($ East + North $) p($ effort $)$ & 11.53 & 0.00 & 14 & 1431.25 \\
\hline$\Psi 1($ East + North $) \varepsilon(T) \gamma($ Neib+East+North $) p($ effort $)$ & 12.21 & 0.00 & 11 & 1438.05 \\
\hline$\Psi 1($ East + North $) \varepsilon($ East*T+North*T) $\gamma($ East + North $+\mathrm{T}) \mathrm{p}($ effort $)$ & 13.57 & 0.00 & 15 & 1431.25 \\
\hline$\Psi 1($ East + North $) \varepsilon($ East*T+North*T) $\gamma($ East + North*T)p(effort $)$ & 15.31 & 0.00 & 16 & 1430.95 \\
\hline$\Psi 1($ East + North $) \varepsilon($ East*T+North*T) $\gamma($ East*T + North $) p($ effort $)$ & 15.56 & 0.00 & 16 & 1431.20 \\
\hline$\Psi 1($ East + North $) \varepsilon($ East*T+North*T) $\gamma($ North $) p($ effort $)$ & 16.59 & 0.00 & 13 & 1438.36 \\
\hline$\Psi 1($ East+North $) \varepsilon($ East*T+North*T) $\gamma($ East $) p($ effort $)$ & 18.33 & 0.00 & 13 & 1440.09 \\
\hline$\Psi 1($ East+North $) \varepsilon($ East*T+North*T) $\gamma($ North $+\mathrm{T}) \mathrm{p}($ effort $)$ & 18.57 & 0.00 & 14 & 1438.29 \\
\hline$\Psi 1($ East + North $) \varepsilon($ East*T+North*T) $\gamma() p.($ effort $)$ & 19.57 & 0.00 & 12 & 1443.38 \\
\hline$\Psi 1($ East+North $) \varepsilon($ East*T+North*T) $\gamma($ North*T)p(effort) & 20.16 & 0.00 & 15 & 1437.85 \\
\hline$\Psi 1($ East + North $) \varepsilon($ East*T+North*T $) \gamma($ East $+\mathrm{T}) \mathrm{p}($ effort $)$ & 20.31 & 0.00 & 14 & 1440.04 \\
\hline$\Psi 1($ East+North $) \varepsilon(.) \gamma($ Neib+East+North $) p($ effort $)$ & 20.42 & 0.00 & 10 & 1448.28 \\
\hline$\Psi 1($ East + North $) \varepsilon($ East*T+North*T) $\gamma(\mathrm{T}) \mathrm{p}($ effort $)$ & 21.61 & 0.00 & 13 & 1443.37 \\
\hline$\Psi 1($ East + North $) \varepsilon($ East*T+North*T) $\gamma($ East*T)p(effort $)$ & 24.84 & 0.00 & 15 & 1442.53 \\
\hline
\end{tabular}

